\title{
NÍVEL DE SOBRECARGA E QUALIDADE DE VIDA DE CUIDADORES DE INDIVÍDUOS COM NECESSIDADES ESPECIAIS
}

Paloma Pellosi ${ }^{1}$, Thayná Araujo Maiolini Costa ${ }^{1}$, Débora Vasconcelos Veríssimo ${ }^{1}$, Thiago Kendy Kawano ${ }^{1}$, Sandra Silva Lustosa ${ }^{2}$, Regina Lucia Meireles Gonçalves Ito ${ }^{3}$, Ana Paula Coelho Figueira Freire ${ }^{1}$, Deborah Cristina Gonçalves Luiz Fernani ${ }^{1,4}$, Francis Lopes Pacagnelli ${ }^{1}$, Carlos Bandeira de Mello Monteiro ${ }^{4,5}$, Maria Tereza Artero Prado ${ }^{1,4}$.

Universidade do Oeste Paulista - UNOESTE, ${ }^{1}$ Curso de Fisioterapia, ${ }^{2}$ Curso de Fonoaudiologia, ${ }^{3}$ Curso de Psicologia, Presidente Prudente, SP. ${ }^{4}$ Faculdade de Medicina do ABC - FMABC, Laboratório de Delineamento de Estudos e Escrita Científica, Santo André, SP. ${ }^{5}$ Universidade de São Paulo - USP, Escola de Artes, Ciências e Humanidades - EACH, Curso de Educação Física e Saúde, São Paulo, SP. e-mail: mariatereza@unoeste.br

\section{RESUMO}

O objetivo desse estudo foi verificar o nível de sobrecarga, a qualidade de vida e a presença de dor em cuidadores de indivíduos com necessidades especiais. Foram entrevistados 40 cuidadores (média de idade de 46,3 $\pm 13,35$ anos) divididos em: Grupo de cuidadores de crianças e adolescentes (GCA) ( $n=18$ ) e Grupo de cuidadores de adultos e idosos (GAI) ( $n=22)$. Na entrevista foram utilizados: questionário inicial para o perfil do cuidador, Questionário de Sobrecarga Burden Interview e Questionário Genérico de Qualidade de Vida SF-36. Foi verificado que $92,5 \%$ dos cuidadores eram mulheres, sendo prevalentes as mães dos indivíduos com necessidades especiais e que $50 \%$ da amostra dedicava-se em tempo integral aos cuidados. Foi verificada a presença de sobrecarga física e emocional de leve à moderada em ambos os grupos, além do acometimento da qualidade de vida, sendo que o GAl apresentou pontuações inferiores aos do GCA em todos os domínios.

Palavras-chave: cuidadores, qualidade de vida, pessoas com deficiência, dor, efeitos psicossociais da doença.

\section{QUALITY OF LIFE AND BURDEN LEVEL OF CAREGIVERS OF INDIVIDUALS WITH SPECIAL NEEDS}

\section{ABSTRACT}

The aim of this study was to verify the level of overload, the quality of life and the presence of pain in caregivers of individuals with special needs. The 40 caregivers (mean age $46.3 \pm 13.35$ years) were interviewed and divided into: Caregivers of children and adolescents group (GCA) $(n=18)$ and Caregivers of adults and elderly group (GAI) ( $n=22)$. In the interview was used: initial questionnaire to caregiver profile, Burden Interview Scale and Quality of Life generic questionnaire SF-36. The caregivers were $92.5 \%$ women, with prevalence of mothers of individuals with special needs, and $50 \%$ engaging in full-time care. It was verified the presence of physical and emotional burden of mild to moderate in both the groups, in addition to impairment of quality of life, being that and GAl showed scores lower than those of the GCA in all areas. Keywords: caregivers, quality of life, disabled persons, pain, cost of illness.

\section{INTRODUÇÃO}

As pessoas com necessidades especiais constituem um grupo heterogêneo, com afecções crônicas (como sequelas neurológicas, distúrbios comportamentais e demências) e vulnerabilidade social $^{1}$. Em decorrência destas afecções, esses indivíduos necessitam constantemente de auxílio, de cuidados básicos e intervenções relacionadas à saúde e a educação, que frequentemente é realizado por um cuidador, sujeito que disponibiliza do tempo, atenção e dedicação parcial ou total com os cuidados destas pessoas $^{1,2}$. Mais de $90 \%$ dos cuidadores são mulheres, sendo esta função desempenhada na maioria das vezes por mães, filhas ou esposas ${ }^{3,4}$. 
Os cuidadores dispõem de grande parte do seu dia às atividades contínuas, que geralmente é realizada no domicílio pela necessidade de cuidado em tempo integral, impossibilidade de dividir o cuidado com outra pessoa, fato que pode acarretar no sentimento de impotência frente à situação do indivíduo cuidado e o surgimento de conflitos familiares ${ }^{5}$. Assim, estes podem ficar expostos a consequências como: desgaste físico, emocional ou psicológico, sendo geralmente decorrente da sobrecarga que é submetido e muitas vezes até acabam por esquecer-se de si próprios e dos outros membros da família ${ }^{1,6}$.

A associação destas consequências pode prejudicar a qualidade de vida (QV) do cuidador, que foi definida pela Organização Mundial da Saúde como a percepção do indivíduo sobre sua posição na vida no contexto da cultura e sistema de valores nos quais ele vive e em relação aos seus objetivos, expectativas, padrões e preocupações ${ }^{7}$. Vale ressaltar que fatores como lazer, saúde e transporte também influenciam na $\mathrm{QV}^{8}$. Deste modo, algumas pesquisas apontam esta redução da QV em cuidadores de indivíduos com lesão medular, paralisia cerebral e transtorno do espectro autístico ${ }^{8-11}$.

A sobrecarga e a alteração da QV do cuidador podem consequentemente afetar a qualidade dos cuidados prestados e até mesmo comprometer a saúde da pessoa que recebe os cuidados $^{12}$, assim justifica-se a importância da realização de estudos que avaliam a sobrecarga e a QV dos cuidadores, para a elaboração de futuros programas educacionais que possam orientar e intervir para propor melhoras nas condições de vida e saúde dos cuidadores.

Deste modo o objetivo deste estudo foi verificar o nível de sobrecarga, a qualidade de vida e a presença de dor em cuidadores de indivíduos com necessidades especiais.

\section{METODOLOGIA}

Estudo transversal aprovado por Comitê de Ética em Pesquisa sob o número do CAAE: 31650214.6.0000.5515, no qual participaram 40 cuidadores ( 37 do sexo feminino e 3 do masculino, com média de idade de 46,3 $\pm 13,35$ anos) de indivíduos com necessidades especiais (com sequelas neurológicas, distúrbios psicomotores e intelectuais) que frequentavam serviços de fisioterapia e reabilitação equestre em uma cidade do oeste paulista. Os cuidadores foram divididos em dois grupos: Grupo de cuidadores de crianças e adolescentes (GCA) com 18 indivíduos e Grupo de cuidadores de indivíduos adultos e idosos (GAI) composto por 22 indivíduos.

Os critérios de inclusão deste estudo foram: a autorização dos indivíduos com a assinatura do termo de consentimento livre e esclarecido e exercer a atividade de cuidador a pelo menos três meses. $E$ a não permanecia frequentemente com o paciente (em torno de 5 vezes na semana) foi o critério de exclusão.

A entrevista com o cuidador foi realizada individualmente, por 2 entrevistadores e em local adequado, com os questionários: Questionário Inicial, Questionário de sobrecarga Burden Inteview ${ }^{13}$ e Questionário Genérico de Qualidade de Vida SF- $36^{14}$.

O questionário inicial foi confeccionado pelos autores e abordava questões a respeito do perfil do cuidador (sexo, idade, escolaridade, renda familiar, presença de parentesco com o indivíduo cuidado), realização da tarefa (tempo que realizava esta atividade e com que frequência) e a presença de dor (sua frequência, intensidade e região acometida).

A versão brasileira do Questionário de Sobrecarga Burden Inteview (BI), traduzido e validado para o português por Scazufca $(2002)^{13}$, é uma escala de 22 itens que verifica a sobrecarga física e emocional do cuidador. Estes itens abrangem as áreas: saúde, vida pessoal, social, emocional e as relações interpessoais, o último item da escala avalia o quanto o cuidador se sente sobrecarregado por cuidar do paciente. Nesta escala é possível realizar a classificação do escore total, no qual a pontuação menor que 21 corresponde a ausência de sobrecarga ou sobrecarga mínima; de 21 a 40: sobrecarga leve a moderada; de 41 a 60: sobrecarga moderada a severa e de 61 a 88: sobrecarga severa.

A aplicação do Questionário Genérico de Qualidade de vida SF-36 elaborado por Ciconelli et al. ${ }^{14}$, quantifica a QV, mede aspectos de saúde e atividades geralmente afetadas pelas condições e estado de saúde. Este questionário é composto por 36 itens, subdivididos em oito domínios: capacidade funcional, aspectos físicos, aspectos emocionais, aspectos sociais, saúde mental, dor, vitalidade, estado geral da saúde e mais um item que compara o estado de saúde atual e no ano anterior. Os escores obtidos em cada domínio são transformados em valores de 0 a 100, onde 0 significa um nível mínimo e 100 um valor máximo atribuído à QV. 
Para análise de normalidade dos dados foi realizado o teste de Shapiro Wilk. Para comparação de variáveis numéricas foi utilizado o teste $\mathrm{t}$ de Student não pareado ou o teste de Mann Whitney, de acordo com a normalidade dos dados. Para o teste qui-quadrado, a correção de Yates foi aplicada em tabelas de contingência $2 \times 2$. Nos demais tipos de configuração de tabela, adotou-se o qui-quadrado para tendência linear. Os dados foram expressos em média e desvio padrão ou mediana e intervalo interquartílico de $25-75 \%$, de acordo com a normalidade dos dados. O nível de significância utilizado foi de $5 \%$.

\section{RESULTADOS}

Os cuidadores avaliados neste estudo são na maioria do sexo feminino (92,5\%) (Tabela 1), com maior prevalência das mães $(83,3 \%)$ no GCA e $(27,3 \%)$ no GAl. O tempo diário que estes dispõem com a atividade de cuidar é em $50 \%$ da amostra o dia inteiro e durante todos os dias da semana (87,5\%). Vale ressaltar que o GCA (11 cuidadores; $61,1 \%$ ) apresentou tempo diário de cuidados superior ao do GAl (9 cuidadores; 40,9\%).

Os dados acerca do nível de escolaridade e da renda familiar mensal estão apresentados na Tabela 1, na qual se observa que a maioria $(37,5 \%)$ dos indivíduos apresentaram ensino médio completo e renda familiar mensal inferior a $R \$ 2000,00$ (90\%), sem diferença significativa entre essas variáveis. Também pode ser observado na Tabela 1, que o GAl apresentou idade superior em relação ao GCA $(p=0,0081)$, sendo que a maioria $(47,5 \%)$ dos cuidadores apresentam idade entre 40 a 60 anos.

Tabela 1. Caracterização dos cuidadores. Dados expressos em média e desvio padrão e em números absolutos e porcentagens, de acordo com a normalidade dos dados.

\begin{tabular}{lccc}
\hline & GCA & GAI & Valor de $\boldsymbol{p}$ \\
\hline Sexo (M/F) & $(1 / 17)$ & $(2 / 20)$ & 0,8564 \\
Idade (anos) & $40,28 \pm 8,36$ & $51,23 \pm 14,77$ & $0,0081^{*}$ \\
Atividade Laboral (Sim/Não) & $(5 / 13)$ & $(10 / 12)$ & 0,4119 \\
Escolaridade & & & \\
$\quad$ Ensino fundamental completo & $3(16,66)$ & $3(13,63)$ & \\
$\quad$ Ensino fundamental incompleto & $3(16,66)$ & $6(27,27)$ & \\
$\quad$ Ensino médio completo & $7(38,88)$ & $8(36,36)$ & 0,4520 \\
$\quad$ Ensino médio incompleto & $2(11,11)$ & $0(0)$ & \\
$\quad$ Ensino superior completo & $3(16,66)$ & $3(13,63)$ & \\
$\quad$ Ensino superior incompleto & $0(0)$ & $2(9,09)$ & \\
Renda familiar mensal & & & \\
$\quad$ Até R\$ 1000,00 & $7(38,88)$ & $6(27,27)$ & \\
$\quad$ De R\$ 1000,00 até R\$ 2000,00 & $11(61,11)$ & $12(54,54)$ & 0,1530 \\
Acima de R\$ 2000.00 & $0(0)$ & $4(18,18)$ & \\
\hline
\end{tabular}

GCA: grupo de cuidadores de crianças e adolescentes; GAl: grupo de cuidadores de adultos e idosos; M: masculino; F: feminino.

Na Tabela 2 visualiza-se os achados da BI e a presença de dor nos grupos analisados. Verificou-se que a média de pontuação obtida pelos cuidadores no $\mathrm{BI}$ compreende entre os escores de 21 a 40 pontos, o que os classifica com sobrecarga leve à moderada sem diferença significativa entre os grupos. Observou-se que 28 cuidadores (70\%) apresentaram dor, sendo 13 indivíduos do GCA e 15 do GAI, porém não houve diferença significativa entre os grupos. Também foi verificado que dentre os cuidadores que apresentaram dor, a maioria dos indivíduos $(71,4 \%)$ possuíam esta em mais de um local do corpo. 
Tabela 2. Escore do nível de sobrecarga e a presença de dor em cuidadores. Dados expressos em média e desvio padrão e números absolutos.

\begin{tabular}{cccc}
\hline & GCA & GAI & Valor de $\boldsymbol{p}$ \\
\hline BI & $31,22 \pm 16,53$ & $29,68 \pm 15,92$ & 0,7663 \\
Presença de dor (Sim/Não) & $(13 / 5)$ & $(15 / 7)$ & 0,9447 \\
\hline
\end{tabular}

GCA: grupo de cuidadores de crianças e adolescentes; GAl: grupo de cuidadores de adultos e idosos; BI: Burden Interview (escore).

Na verificação da QV foi observado baixas pontuações em ambos os grupos. No GCA os domínios de vitalidade e dor apresentaram os valores mais inferiores, já no GAl foram os domínios de vitalidade, aspectos físicos e aspectos emocionais, sendo que apenas neste último domínio houve diferença significativa $(p=0,0386)$ entre os grupos analisados (Tabela 3$)$.

Tabela 3. Escores dos domínios do Questionário de Qualidade de Vida (SF-36) em cuidadores de ambos os grupos. Valores expressos em mediana e intervalo interquartílico de $25-75 \%$.

\begin{tabular}{lccc}
\hline & GCA & GAI & Valor de $\boldsymbol{p}$ \\
\hline Capacidade Funcional & $90,0[72,50-96,25]$ & $80,0[70,00-95,00]$ & 0,5831 \\
Aspectos Físicos & $75,0[50,00-100,0]$ & $50,0[18,75-81,25]$ & 0,1347 \\
Dor & $67,0[38,50-100,0]$ & $51,0[38,50-88,00]$ & 0,3484 \\
Estado Geral de Saúde & $77,0[67,00-88,25]$ & $72,0[53,75-87,75]$ & 0,6918 \\
Vitalidade & $65,0[40,00-76,25]$ & $40,0[33,75-71,25]$ & 0,1467 \\
Aspectos Sociais & $87,5[62,50-100,0]$ & $75,0[50,00-90,63]$ & 0,1407 \\
Aspectos Emocionais & $100,0[58,35-100,0]$ & $50,0[0,0-100,0]$ & $0,0386 *$ \\
Saúde Mental & $74,0[61,00-85,00]$ & $58,0[47,00-81,00]$ & 0,2303 \\
\hline
\end{tabular}

GCA: grupo de cuidadores de crianças e adolescentes; GAl: grupo de cuidadores de adultos e idosos.

\section{DISCUSSÃO}

Foi verificada a presença de sobrecarga física e emocional de leve à moderada em ambos os grupos avaliados, porém mesmo esta não sendo expressiva, há o acometimento da QV destes indivíduos, evidenciada por baixas pontuações no questionário de QV. Além disso, vale ressaltar que o GAl apresentou pontuações inferiores aos do GCA em todos os domínios da avaliação da $Q V$, fato que pode demonstrar o maior acometimento da QV neste grupo.

Os cuidadores avaliados neste estudo são na maioria do sexo feminino, com maior prevalência das mães, geralmente com dedicação em tempo integral e em todos os dias da semana. A maioria dos cuidadores apresentaram ensino médio completo e renda familiar mensal inferior a 3 salários mínimos. 0 estudo de Ferreira et al. ${ }^{15}$, avaliaram o perfil de 40 cuidadores de idosos e verificaram que a maioria era do sexo feminino com prevalência das filhas (50\%) na realização dos cuidados. A maior parte da amostra (55\%) apresentava apenas o ensino fundamental, renda familiar mensal de 1 a 3 salários mínimos $(62,5 \%)$ e $67,5 \%$ dos cuidadores se dedicavam integralmente ao cuidado (de 18 a 24 horas por dia) e em todos os dias da semana. Deste modo, observa-se a intensa dedicação dos cuidadores à realização dos cuidados, que muitas vezes acabam por abdicar da possibilidade de inclusão no mercado de trabalho, de aprimorar seus estudos, de realizar atividades de lazer e até mesmo de autocuidado, que reflete negativamente na saúde, bem-estar e convívio social desses cuidadores em consequência ao desgaste físico e emocional a que esses indivíduos são submetidos diariamente ${ }^{1}$.

Nesse estudo foi observado que 28 cuidadores (70\%) relataram sentir dor em mais de um local do corpo, sendo que a presença desta, também foi evidenciada no estudo de Almeida e Conceição ${ }^{16}$, no qual os cuidadores foram avaliados pelo questionário de dor de $\mathrm{McGill}^{17}$, sendo observado a presença de dor na 
coluna cervical $(46,7 \%)$, ombro $(46,7 \%)$ e na coluna lombar $(66,7 \%)$. Tal fato pode ser causado em função das atividades de cuidado, pois estas são geralmente contínuas e intensas, além da possibilidade de serem executadas de forma inadequada, como a manutenção de posturas e realização de transferências de forma incorreta durante a prestação de cuidados ao longo do dia, o que pode desencadear agravos à saúde física desses cuidadores. Em contrapartida, no estudo de Orsini et al. ${ }^{18}$, que avaliou 9 cuidadores de indivíduos com esclerose lateral amiotrófica sob tratamento em um serviço de neurologia hospitalar, verificou que apenas um dos cuidadores relatava a presença de quadro álgico na coluna lombar.

Em relação ao nível de sobrecarga, os cuidadores foram classificados pela $\mathrm{BI}$ com sobrecarga leve à moderada (GCA: $31,22 \pm 16,53$ pontos e GAl: $29,68 \pm 15,92$ pontos). No estudo de Stackfleth et al. ${ }^{19}$, que avaliaram 60 cuidadores de idosos fragilizados pelo mesmo instrumento de avaliação utilizado nesta pesquisa, foi encontrada uma média de pontuação inferior $(24,48 \pm 13,6)$ comparada a deste estudo, no entanto os indivíduos também foram classificados, com sobrecarga leve à moderada. Outro estudo ${ }^{16}$, analisou a sobrecarga de 15 cuidadores de crianças com paralisia cerebral, sendo verificado que destes, 9 indivíduos (60\%) apresentaram sobrecarga moderada, segundo a Escala de Sobrecarga do Cuidador de Zarit $^{20}$. Assim, o achado acerca do nível de sobrecarga, pode ser justificado pelo fato de que a maioria dos cuidadores geralmente são as mães, que acreditam ser as únicas capazes a proporcionar ao seu filho uma atenção incondicional $^{21}$, além de pensar que a atividade de cuidar seja parte de suas obrigações familiares e assim não encaram essa tarefa como uma sobrecarga.

Na verificação da $Q V$, o GAl apresentou pontuações inferiores aos do GCA em todos os domínios, sendo observado que no GCA os domínios de vitalidade e dor foram os que apresentaram os menores valores e no GAI foram os domínios de vitalidade, aspectos físicos e aspectos emocionais. Esses achados vão de encontro aos obtidos em outro estudo ${ }^{22}$, que avaliaram 12 cuidadores de indivíduos com disfunções neurológicas sob tratamento em uma clínica de fisioterapia pelo questionário de qualidade de vida SF-36, no qual foi observado os resultados pré e pós intervenção fisioterapêutica, sendo visto que os domínios que apresentaram valores mais baixos foram: aspectos emocionais, aspectos físicos e vitalidade e no domínio de dor observou-se uma melhora significativa após as orientações fisioterápicas. Resultados similares também foram encontrados no estudo de Ferreira et al. ${ }^{15}$, que avaliaram cuidadores de idosos e foi visto que os domínios que apresentaram valores inferiores foram: aspectos emocionais, vitalidade e dor.

Estas pontuações inferiores do GAI comparado ao GCA na QV, pode ser explicado pelo fato que no GCA, a mãe desde o nascimento do filho sabe que este será dependente dela, desta forma torna-se capaz de organizar a estrutura e funcionamento familiar, para realizar esta função de cuidar ${ }^{23}$. O maior acometimento da QV no GAl, pode ser justificado pelo fato de que o indivíduo que recebe os cuidados torna-se dependente de auxílio para realização das atividades básicas de vida diária de uma hora para outra, o que pode impossibilitar esta organização familiar, ou então em decorrência destes indivíduos receberem cuidados a muito tempo, assim os cuidadores apresentam maiores possibilidades do surgimento de alterações de ordem emocional, física e social ${ }^{24}$.

\section{CONCLUSÃO}

Os cuidadores de ambos os grupos foram predominantemente mulheres, com dedicação integral aos cuidados, com baixa renda familiar e queixa de dor. Apresentaram sobrecarga de leve à moderada e acometimento da $\mathrm{QV}$, sendo esta maior no GAl.

Deste modo, faz-se necessário a elaboração de programas educacionais de orientação fisioterapêutica aos cuidadores, para ensiná-los como é a postura correta para execução da atividade de cuidar, a forma adequada para realizar a manipulação e transferência do indivíduo com necessidades especiais. Com o intuito de prevenir o desencadeamento da dor, o surgimento de sobrecarga e até mesmo o comprometimento da QV. Porém, quando a dor estiver presente, o cuidador deverá ser inserido em um programa de reabilitação fisioterapêutica para amenizar e/ou eliminar esta. Além disso, o fisioterapeuta deverá enfatizar sobre a importância de o indivíduo ter momentos de descanso, de prática de atividade física, de lazer e de cuidar de si próprio, para evitar complicações decorrentes, como a sobrecarga emocional. 


\section{CONFLITO DE INTERESSE}

Os autores declaram não haver qualquer potencial de conflito de interesse que possa interferir na imparcialidade deste trabalho científico.

\section{REFERÊNCIAS}

1. Braccialli LMP, Bagagi PS, Sankako AN, Araújo RCT. Qualidade de vida de cuidadores de pessoas com necessidades especiais. Rev Bras Educ Espec. 2012;18(1):113-26.

DOI:

http://dx.doi.org/10.1590/S1413-

\section{8}

2. Marcelino CAG, Cruz DM, Rueda LJ. The efficacy of telephone use to assist and improve the wellbeing of family caregivers of persons with chronic diseases: a systematic review protocol. JBI Database System Rev Implement Rep. 2013;11(2):330-42.

DOI: http://dx.doi.org/10.15124/CRD42013004248

3. Yamashita $\mathrm{CH}$, Amendola F, Alvarenga MRM, Oliveira MAC. Perfil sociodemográfico de cuidadores familiares de pacientes dependentes atendidos por uma unidade de saúde da família no município de São Paulo. O Mundo da Saúde. 2010;24(1):20-4.

4. Trigueiro LCL, Lucena NMG, Aragão POR, Lemos MTM. Perfil sociodemográfico e índice de qualidade de vida de cuidadores de pessoas com deficiência física. Fisioter Pesqui. 2011;18(3):2237. DOI: http://dx.doi.org/10.1590/S180929502011000300004

5. Bochi SCM. Vivenciando a sobrecarga ao vir-aser um cuidador familiar de pessoa com acidente vascular cerebral (AVC): uma análise do conhecimento. Rev Latino-Am Enferm. 2004;12(1):115-21.

DOI:

http://dx.doi.org/10.1590/S0104-

\section{6}

6. Valente LE, Truzzi A, Souza WF, Alves GS, Alves CEO, Sudo FK et al. Helth self-perception by dementia family caregivers sociodemographic and clinical factors. Arq Neuro-Psiquiatr. 2011;69(5):739-44

DOI:

http://dx.doi.org/10.1590/S0004-

\section{X2011000600003}

7. The World Health Organization Quality of Life assessment (WHOQOL): position paper from the World Health Organization. Soc Sci Med. 1995;41(10):1403-9.

8. Barbosa MRP, Fernandes FD. Qualidade de vida dos cuidadores de crianças com transtorno do espectro autístico. Rev Soc Bras Fonoaudiol.
2009;14(4):482-6.

DOI:

http://dx.doi.org/10.1590/S1516-

\section{9}

9. Graça A, Nascimento MA, Lavado EL, Garanhani MR. Quality of life of primary caregivers of spinal cord injury survivors. Rev Bras Enferm. 2013;66(1):79-83.

10. Nogueira PC, Rabeh SAN, Caliri MHL, Dantas RAS, Haas VJ. Burden of care and its impact on health-related quality of life of caregivers of individuals with spinal cord injury. Rev Latino-Am Enferm. 2012;20(6):1048-56. DOI: http://dx.doi.org/10.1590/S010411692012000600006

11. Zanon MA, Batista NA. Quality of life and level of anxiety and depression in caregivers of children with cerebral palsy. Rev Paul Pediatr. 2012;30(3):392-6.

DOI:

http://dx.doi.org/10.1590/S0100305822012000300013

12. A'Campo LEI, Spliethoff-Kamminga NG, A, Macht M. Caregiver education in Parkinson's disease: formative evaluation of standardized program in seven European countries. Qual Life Res. 2010;19(1):55-64. DOI: http://dx.doi.org/10.1007/s11136-009-9559-y

13. Scazufca M. Brazilian version of the Burden Interview scale for the assessment of burden of care in carers of people with mental illnesses. Rev Bras Psiquiatr. 2002;24(1):12-7. DOI: http://dx.doi.org/10.1590/S151644462002000100006

14. Ciconelli RM, Ferraz MB, Santos W, Meinão I, Quaresma MR. Tradução para a língua portuguesa e validação do questionário de qualidade de vida SF-36 (Brasil SF-36). Rev Bras Reumatol. 1999;39(3):143-50.

15. Ferreira CG, Alexandre TS, Lemos ND. Fatores associados à qualidade de vida de cuidadores de idosos em assistência dominiliária. Saude Soc. 2011;20(2):398-409.

DOI:

http://dx.doi.org/10.1590/S010412902011000200012

16. Almeida MS, Conceição TMA. Prevalência de sintomas álgicos, sobrecarga e qualidade de vida de cuidadores de crianças com paralisia cerebral. Rev Pesq Fisiot. 2013;3(1):36-49. DOI: http://dx.doi.org/10.17267/2238-

\section{4rpf.v3i1.143}

17. Pimenta CAM, Teixeira MJ. Questionário de dor McGill: proposta de adaptação para a língua portuguesa. Rev Esc Enferm USP. 1996;30(3):47383. DOI: http://dx.doi.org/10.1590/S0080$\underline{62341996000300009}$ 
18. Orsini $M$, Mello $M$, Lisieux D, Pássaro $C P$, Leite MAA, Baldez AC et al. Qualidade de Vida de Cuidadores e Pacientes com Diagnóstico de Esclerose Lateral Amiotrófica. Rev Neurocienc 2012;20(2):215-21.

19. Stackfleth R, Diniz MA, Fhon JRS, Vendruscolo TRP, Fabrício-Whebe SCC, Marques S. Sobrecarga de trabalho em cuidadores de idosos fragilizados que vivem no domicílio. Acta Paul Enferm. 2012;25(5):768-74.

DOI:

http://dx.doi.org/10.1590/S0103-

21002012000500019

20. Sequeira CAC. Adaptação e validação da escala de sobrecarga do cuidador de Zarit. Rev Enf Ref. 2010;2(12):9-16.

21. Pinto MB, Assis FAG, Santos NCCB, Torquato $\mathrm{IMB}$, Collet N. Significado do cuidado à criança deficiente com necessidades especiais: relato de mães. Ciênc Cuid Saude. 2014;13(3):549-55. DOI: http://dx.doi.org/10.4025/cienccuidsaude.v13i3.

\section{$\underline{22189}$}

22. Fernandes BCW, Ferreira KCP, Marodin MF, do Val MON, Fréz AR. Influência das orientações fisioterapêuticas na qualidade de vida e na sobrecarga de cuidadores. Fisioter Mov. 2013;26(1):151-8. DOI: http://dx.doi.org/10.1590/S0103-

51502013000100017

23. Sanchez LAS, Júnior GAF. Relatos maternos sobre o impacto do diagnóstico da deficiência dos filhos. Cad Saúde Colet. 2011;19(3):366-74.

24. Guedea MTD. Modelo de estresse e bemestar subjetivo em cuidadores de familiares idosos dependentes funcionais [tese]. Brasília: Universidade Nacional de Brasília; 2005.

Recebido para publicação em 27/07/2016

Revisado em 19/08/2016

Aceito em 05/09/2016 